\title{
ARTÍCULO EDITORIAL
}

\section{SISTEMAS DE NANOSENSORES PARA MATERIALES INTELIGENTES}

En el Centro Nacional de Investigaciones Metalúrgicas (CENIM), integrado en el CSIC (www.csic.es) de España, un grupo de investigadores trabaja en la investigación y desarrollo de una familia de nanosensores (elementos muy pequeños captadores de variaciones en el material y desarrollados con nanotecnologías), los que se instalan embebidos en materiales no metálicos, con la finalidad de obtener información sobre el comportamiento de los mismos, ante diversos estímulos exteriores. Luego, esta información se transmite a un sistema encargado de la gestión de estos eventos. Esta tecnología, pionera en los denominados "materiales inteligentes" que constituyen una innovación importante en la ciencia de materiales, abre la posibilidad de tener información en tiempo real sobre el comportamiento del material ante diversas contingencias no previstas.

En el presente artículo se describe el desarrollo que está teniendo esta investigación dentro de nuestro grupo de trabajo, los campos de aplicación de la misma en ingeniería de materiales, así como los objetivos que persigue, logros obtenidos y las posibles fuentes de financiamiento para continuar la investigación en el futuro próximo, y las áreas en que se esperan mayores impactos de los resultados.

Muchos materiales tradicionales, de composición metálica, están siendo sustituidos por materiales fabricados con "composites" y resinas. En el interior de este tipo de materiales se hace posible instalar nanosensores que informen de lo que ocurre en el material en caso de fatiga, colisión, variaciones extremas de temperatura, etc.

\section{Antecedentes}

Los materiales metálicos pueden ser sustituidos ventajosamente por materiales sintéticos de las mismas características físicas, pero con ventajas mecánicas y económicas, que serán comentadas más adelante. Esta sustitución, que se viene haciendo desde hace años, constituye una tendencia que continuará desarrollándose en los próximos años. Nuestra innovación en este campo consiste en dotar al material de inteligencia, de acuerdo con la descripción del presente artículo.

\section{Nuevos materiales compuestos}

Nuestro interés está orientado a la producción industrial de nuevos materiales inteligentes aplicados - en principio - a los campos de defensa, vehículos y aeronaves, campo que posteriormente podría extenderse a otras áreas de aplicación industrial.

Nos centramos en la investigación y desarrollo de estructuras de materiales de tipo "sándwich", que se realizan sobre la base de "composites", con una malla metálica central que otorga consistencia a la pieza. El "composite" es casi todo el conjunto de lo que denominaremos "estructura", que corresponde a la pieza dotada de todos sus componentes. La estructura queda terminada con unos revestimientos metálicos. En la Figura 1 se observa la composición de estas estructuras de tipo "sándwich", con una malla metálica central, embebida en el "composite"; ésta constituye la base de nuestras investigaciones en el área de los materiales compuestos.

En estas estructuras de nuevos materiales se instalará un conjunto de nanosensores y un dispositivo electrónico, con el propósito de obtener datos generados por los sensores, que permitirán saber en todo momento qué sucede en el material, ante cualquier contingencia.

\section{Componentes del sistema de sensores}

Para el equipo de investigadores, este sistema introduce una innovación importante en los nuevos materiales inteligentes y tiene la siguiente filosofía, en cuanto a los componentes utilizados en el mismo:

- Nanosensores de tipo elástico, que captan deformaciones y las transmiten a un microsistema óptico.

- Nanosensores de tipo sísmico, para captar golpes y vibraciones. 
- Utilización de un sistema microelectrónico para transmitir datos.

- Utilización de nanofibras ópticas para transmitir datos.

Estos elementos se encuentran todos embebidos en el propio material "composite".

Externamente, existe un receptor de los datos que también realiza su procesado para poder dar información coherente al usuario.

\section{Estado del arte}

En este apartado se describe de manera sucinta el actual nivel de desarrollo en esta área de conocimiento, desde diferentes industrias y centros de investigación.

Las industrias de fabricación de autos, aeronáutica y de construcción, están investigando desde hace tiempo la utilización de nuevos materiales que sustituyan, aun de una manera parcial, a los tradicionales materiales metálicos. Estos nuevos materiales presentan un conjunto de ventajas, como los citados a continuación:

- Menor peso que los materiales tradicionales.

- Menor coste que otros materiales de uso común.

- Mayor resistencia que otros materiales.

- Mayor facilidad para el reciclaje del material al finalizar su vida útil, con la ventaja de poder reciclar de una manera sencilla y cumplir la normativa de la Unión Europea (UE), que obliga a partir de 2.015 a reciclar el $95 \%$ del material desechable, al finalizar la vida útil del mismo.

En Francia existen proyectos de este tipo para la industria naval, en Estados Unidos para la industria aeronáutica y en España para la industria del automóvil. Esto demuestra el interés industrial que despiertan este tipo de materiales y su aplicación.

En estos momentos se está consiguiendo esta sustitución de manera paulatina, pero se espera añadir mejoras en determinadas aplicaciones, que transformen al material en inteligente y aumenten las ventajas de utilizar estos materiales de manera intensiva.

Este tipo de mejoras, adaptadas siempre a la utilización posterior del material, constituye objeto de investigación , dada la importancia que su aplicación reviste en el área de la ingeniería, en cada uno de los campos en que se emplea.

La base de nuestras actuales investigaciones se puede encontrar en la consecución de los siguientes puntos:

- El desarrollo de placas laminadas con mallas laminadas, basadas en la teoría de la deformación.

- Algoritmos de simulación para medir la resistencia del material.

- Test de fatiga.

- Fabricación ecológica.

\section{Objetivos}

Nuestra investigación se propone lograr los siguientes objetivos:

- Investigar estructuras de materiales "composites" que contienen en su interior una malla metálica para dar resistencia al material. Esa malla puede ser de cobre, acero, aluminio o de alguna aleación, según la aplicación a la que se destina el material en cuestión y su composición, que es una parte de la investigación.

- Determinar cuáles son las láminas metálicas más adecuadas para recubrir las estructuras en ambas caras, que son las que les proporcionan su especial terminado.

- Investigar y desarrollar un conjunto de nanosensores para ser instalados, embebidos dentro del material, para adquirir datos, con toda la electrónica asociada y las nanofibras ópticas que permiten la transmisión de los datos.

- Desarrollar un microtransmisor embebido, encargado de enviar los datos al exterior de la estructura. 


\section{Estructuras de material}

Las estructuras que se investigan son tipo sándwich, como ya se ha señalado, de aspecto semejante al de las Figuras 1 (malla utilizada) y 2.

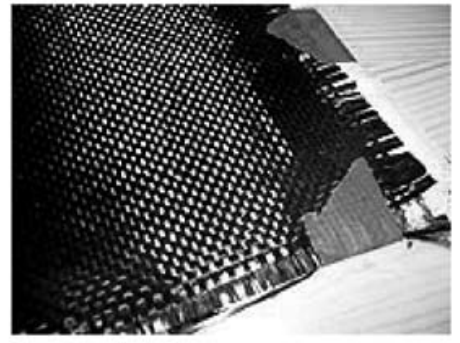

Figura 1. Malla interior

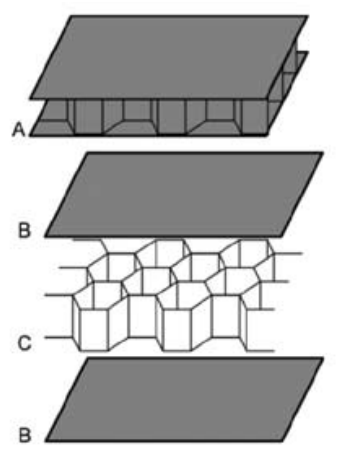

Figura 2. Estructura sandwich

En la Figura 1 se puede ver una malla metálica, de las utilizadas en nuestras investigaciones. En la Figura 2 se tiene el esquema práctico de cómo se realiza una estructura de "composites" con una malla metálica en forma de panel de abeja.

Sobre este tipo de estructuras desarrollamos nuestro trabajo de investigación y tratamos de lograr los objetivos propuestos.

\section{Nanosensores}

Los nanosensores que se instalarán en el interior de la estructura se interconectarán con la utilización de fibras ópticas, que trabajan en transmisión y recepción, es decir, se utilizan dos fibras para interconexión, como se aprecia en la Figura 3.

En la Figura 3 los sensores aparecen señalados como a, b, c, etc., y se unen a una fibra doble por la que transmitirán/ recibirán los datos. Incorporan un nanoconvertidor óptico y envían información con su código. Los sensores son de tipo elástico para detectar deformaciones que pueda sufrir el material o bien de tipo sísmico, para poder detectar impactos y vibraciones a los que se ha sometido el material. Finalmente, la fibra se encuentra con un recolector de datos, que se une al microtransmisor que enviará la información.
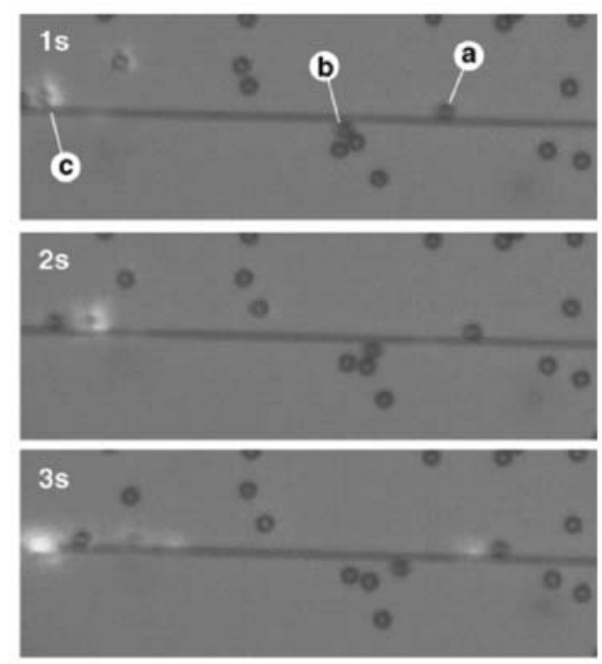

Figura 3. Conexión de nanosensores.

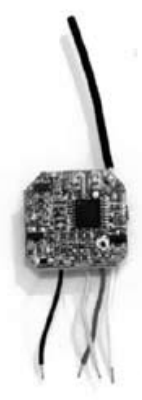

Figura 4. Microtransmisor. 


\section{Microtransmisor}

Todas las fibras se conectarán a un microdispositivo electrónico que tiene como objetivo transmitir estos datos a un sistema exterior, utilizando un microtransmisor, tal como el presentado en la Figura 4. Este microtransmisor aprovecha como antena la cubierta metálica del "sándwich". Las pruebas de transmisión se realizan en FM (frecuencia 2.432 MHz) y en frecuencias compatibles con dispositivos WI-FI (2 a $6 \mathrm{GHz}$ ), para determinar cuál de los sistemas permite más alcance con menor consumo. Buscamos un alcance mínimo de 50 m y deseable de $100 \mathrm{~m}$. Otra línea de investigación, está relacionada con el modo de realizar de manera eficiente el aporte exterior de energía, necesaria para que el sistema funcione correctamente.

\section{Recepción externa}

El receptor transmisor externo es el encargado de recibir los datos, contando con el software desarrollado en el proyecto para tratarlos. Para ello se implementarán algoritmos que permitan predeterminar daños en colisiones, efecto de las temperaturas, vibraciones, etc., a partir de la información procedente de los sensores y de lo que ocurre en el material. Esto es muy importante en las áreas de defensa, aeronáutica, construcción y automoción. El software se desarrolla en lenguaje JAVA.

Básicamente este sistema de recepción, consta de un receptor-transmisor adecuado a la frecuencia que se esté utilizando, y de un microprocesador con memoria para contener el software aplicativo. En un display aparece la información del sistema de forma adaptada a la operatoria propia del controlador. Este sistema externo no debe estar miniaturizado, pues es totalmente exterior al material y de índole convencional.

\section{Conclusiones}

El desarrollo experimental efectuado por nuestro grupo ha sido realizado en fases sucesivas, que se describen a continuación:

1. Obtención de un conjunto de estructuras, aún no inteligentes, realizadas con "composites" y resinas, que sean adecuadas para los fines perseguidos.

2. Investigación y desarrollo de la malla metálica que se utilizará, de forma que permita una consistencia adecuada para la aplicación industrial de la estructura.

3. Investigación y desarrollo de los nanosensores que se utilizarán. Ésta constituye la etapa más delicada, pues los sensores son extremadamente pequeños y deben de tener una vida útil alta, de tal manera de no invalidar su función de forma prematura. Buscamos en principio una vida de un año, tiempo que en el futuro se pretende ampliar. Los nanosensores poseen un código que los identifica y un transductor óptico que permite, cuando son interrogados, que envíen la información a la electrónica de recolección de datos. La conexión de los sensores, con el centro de recolección de datos en el interior del material es multipunto, por fibra óptica, tal como se ha indicado.

4. Selección de las nanofibras ópticas más adecuadas para ser utilizadas en la estructura. Para cada tipo de aplicación industrial, el tipo de fibra óptica utilizada es diferente.

5. Investigación de la electrónica asociada al conjunto de nanosensores, desarrollo de la misma y del software de operación.

6. Investigación y desarrollo del sistema radiante, que consiste en un microtransmisor del tipo ya expuesto y su conexión a la estructura externa para que actúe de antena y pueda enviar los datos al receptor. Se investiga la direccionalidad de este transmisor (para aprovechar la energía al máximo) y la frecuencia más adecuada para realizar la transmisión.

7. Alimentación externa del sistema electrónico. Este punto es muy importante pues se ha de garantizar, de una manera fiable y económica el funcionamiento del sistema embebido.

8. Instalación del conjunto de sensores y electrónica en el material, de la manera más conveniente para el correcto funcionamiento del mismo, en las condiciones más óptimas posibles.

9. Desarrollo de una batería de tests para verificar los resultados obtenidos, en concordancia con las previsiones de la investigación.

Hemos de señalar que todos los procesos se realizan siguiendo un plan de calidad enmarcado en ISO 9000. 


\section{Ejemplo práctico de aplicación experimental}

En este apartado queremos exponer una estructura concreta, realizada de manera experimental, según los métodos descritos, que sirve como elemento de prueba y que posteriormente tendrá una aplicación industrial concreta.

Como ejemplo de lo que hemos realizado, podemos presentar el desarrollo de una estructura aplicada a una UAS (Unmanned Aerial System), también conocido como UAV (Unmanned Aerial Vehicle). Este tipo de vehículos no tripulados, están adquiriendo bastante utilidad en aplicaciones tanto militares como civiles (vigilancia de fronteras). Como equipo de investigación, consideramos que la demanda de estas estructuras en la industria va en aumento, lo que le otorga un interesante valor agregado, razón por la cual nos empeñamos en el desarrollo de la mencionada estructura.

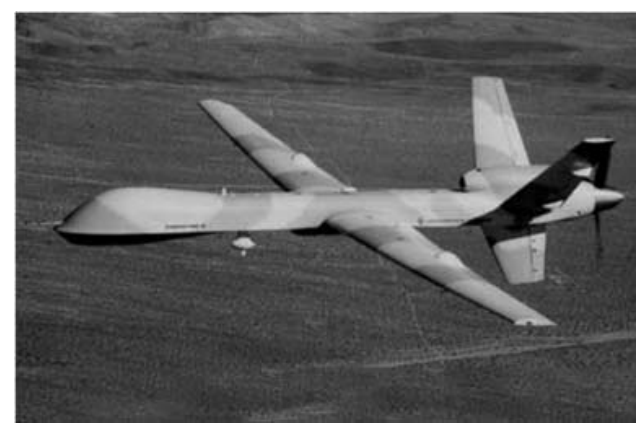

Figura 5. UAS.

En la Figura 5 tenemos un ejemplo de UAV de una envergadura de 1,62 m, que puede servir perfectamente para realizar nuestros ensayos.

Un $80 \%$ de su estructura estaría formada por estructuras del tipo que hemos descrito en los apartados anteriores.

La utilidad de esta UAV se centraría en vigilancia de fronteras, llevando un motor, un sistema de dirección, un compartimento de combustible y otro compartimento con la instrumentación electrónica (video cámaras y captadores de sonido).

En este ensayo la autonomía de la UAV, así como su equipamiento, no constituye parte de nuestro interés. Estudiamos cómo responde el material y de qué manera el sistema de sensores es capaz de obtener datos interesantes, procesarlos y enviarlos en tiempo real al sistema captador de datos.

El sistema es reutilizable. En principio está equipado con cuatro nanosensores sísmicos, cinco elásticos y un sistema óptico de toma de datos. Estos datos son enviados por medio de un transmisor miniatura en FM al exterior, para su tratamiento en el sistema de supervisión. De esta forma sometemos a la estructura a distintos procesos y vemos si el sistema de sensores registra los datos con la debida fidelidad y es capaz de transmitirlos al controlador.

Este modelo sirve muy bien para pasar el banco de tests desarrollados durante la investigación y poder verificar de manera experimental los desarrollos realizados.

\section{Resumen de resultados obtenidos}

Hasta el momento hemos obtenido los siguientes resultados:

- Investigación y desarrollo de estructuras de materiales, suficientes para las pruebas de los sensores.

- Investigación y desarrollo de la primera generación de sensores.

- Investigación y desarrollo del sistema óptico y la fibra que se utilizará.

- Ingeniería de la instalación de los componentes en la estructura.

- Primeras pruebas sobre prototipo

Este equipo de trabajo considera que el $40 \%$ de los objetivos propuestos han sido alcanzados. 


\section{Trabajos futuros}

En el futuro próximo queremos conseguir resultados aplicables a la aeronáutica y a los automóviles, industrias muy importantes, que pueden verse ampliamente beneficiadas con esta tecnología.

Las metas que nos planteamos están relacionadas a conseguir financiamiento para ampliar los objetivos del proyecto e introducirnos en el campo de las aeroestructuras y de la automoción.

\section{Procedimientos de financiamiento}

Para lograr nuestros objetivos, precisamos de mayor financiamiento y de un trabajo cooperativo con otros grupos de investigación. Pretendemos lograr lo anterior mediante un proyecto del VII Programa Marco de la Unión Europea.

Por ello hemos creado un consorcio de socios que trabajan en líneas similares de investigación, presentando a la Unión Europea una propuesta denominada NESBE, con el propósito de solicitar el financiamiento que permita continuar estas investigaciones aplicadas a la industria.

En la siguiente tabla indicamos las instituciones europeas de investigación que participan en este consorcio.

\begin{tabular}{|c|c|c|c|c|}
\hline Número & Organización & País & $\begin{array}{c}\text { Tipo de } \\
\text { organización }\end{array}$ & Siglas \\
\hline 1. Coordinador & $\begin{array}{l}\text { Agencia Estatal Consejo Superior de } \\
\text { Investigaciones Científicas }\end{array}$ & España & $\begin{array}{l}\text { National Research } \\
\text { Center }\end{array}$ & CSIC \\
\hline 2 & Baris Elektrik S.A. & Turquía & SME & BAR \\
\hline 3 & $\begin{array}{l}\text { Technological Institute of Optics, Colour } \\
\text { and Imaging }\end{array}$ & España & Research Center & AIDO \\
\hline 4 & $\begin{array}{l}\text { FADA-CATEC Center for Advanced } \\
\text { Aerospace Technologies }\end{array}$ & España & Research Center & CATEC \\
\hline 5 & Riga Technical University & Letonia & $\begin{array}{l}\text { University-Research } \\
\text { Center }\end{array}$ & RTU \\
\hline 6 & HLP DEVELOPMENT S.L. & Francia & SME & HLP \\
\hline 7 & TROYKA LTD & Turquía & SME & TRO \\
\hline 8 & $\begin{array}{l}\text { University of Chemical Technology and } \\
\text { Metallurgy }\end{array}$ & Bulgaria & $\begin{array}{l}\text { National Research } \\
\text { Center-University }\end{array}$ & UCTM \\
\hline 9 & Tampere University of Technology & Finlandia & $\begin{array}{l}\text { University-Research } \\
\text { Center }\end{array}$ & TUT \\
\hline 10 & Centro Ricerche FIAT S.C.p.A. (CRF) & Italia & Research Center & CRF \\
\hline 11 & SINTEF Group & Noruega & Research Center & SIN \\
\hline
\end{tabular}

\section{Impacto}

Esperamos un impacto importante de estas investigaciones y desarrollos, en las siguientes áreas:

- Industria aeronáutica.

- Industria del automóvil.

- Industria de construcción de buques.

- Ingenierías dedicadas a materiales.

- Ingenierías de Sistemas.

\author{
Dr. Manuel Rincón Arche \\ Científico Titular CENIM-CSIC \\ España \\ E-mail: mrincon@cenim.csic.es
}

\author{
Dr. José Antonio Robla \\ Científico Titular CENIM-CSIC \\ España
}

